\begin{tabular}{|c|c|}
\hline Title & Effects of neurofeedback on the activities of motor-related areas by using motor execution and imagery \\
\hline Author(s) & Y ang, Huixiang; Hu, Zhengfei; Imai, Fumihito; Y ang, Y uxiang; Ogawa, Kenji \\
\hline Citation & $\begin{array}{l}\text { Neuroscience letters, } 746,135653 \\
\text { https://doi.org/10.1016/.neul let.2021.135653 }\end{array}$ \\
\hline Issue Date & 2021-02-16 \\
\hline Doc URL & http:/hdl. handle.net/2115/84163 \\
\hline Rights & $\begin{array}{l}\text { () 2021. This manuscript version is made available under the CC-BY-NC-ND } 4.0 \text { license } \\
\text { http://creativecommons.org/icenses/oy-nc-nd/4.0/ }\end{array}$ \\
\hline Rights(URL) & http://creativecommons.org/icenses/by-nc-nd/4.0/ \\
\hline Type & article (author version) \\
\hline File Information & Manuscript_NSL_final.pdf \\
\hline
\end{tabular}

Instructions for use 


\title{
Effects of neurofeedback on the activities of motor-related areas by using motor execution and imagery
}

\author{
Huixiang Yang, Zhengfei Hu, Fumihito Imai, Yuxiang Yang, Kenji Ogawa* \\ Department of Psychology, Hokkaido University, Sapporo, Japan \\ 5 Correspondence should be addressed to: \\ Dr. Kenji Ogawa, Department of Psychology, Hokkaido University, Kita 10, Nishi 7, \\ Kita-ku, Sapporo, 060-0810 Japan \\ Tel/Fax: +81-011-706-4093 \\ E-mail: ogawa@let.hokudai.ac.jp.
}




\begin{abstract}
Previous studies have reported that real-time functional magnetic resonance imaging (fMRI) neurofeedback using motor imagery can modulate the activity of several motor-related areas. However, the differences in these modulatory effects on distinct motor-related target regions using the same experimental protocol remain unelucidated. This study aimed to compare neurofeedback effects on the primary motor area (M1) and the ventral premotor cortex (PMv). Of the included participants, 15 received blood oxygenation level-dependent (BOLD) signals from their left M1, and the other 15 received signals from their left PMv. Both groups were instructed to try to increase the neurofeedback score (NF-Score), which reflected the averaged activation level of the target region, by executing or imagining a right-hand clenching movement. The result revealed that during imagery condition, the left M1 was deactivated in the PMv-group but not in the M1-group, whereas the left PMv was activated in the PMv-group but not in the M1-group. Our finding indicates that neurofeedback from distinct motor-related regions has different effects on 15 brain activity regulation.
\end{abstract}

\title{
Keywords
}

Neurofeedback; real-time fMRI; motor execution; motor imagery; primary motor cortex; ventral premotor cortex. 


\section{Introduction}

Neurofeedback using functional magnetic resonance imaging (fMRI-nf) allows for online data processing and information display. This helps participants use functional information from the blood oxygenation level-dependent (BOLD) signals from their brain to learn

5 controlling their brain activity (Sulzer et al., 2013). Neurofeedback for motor-related regions has the potential to achieve primary goals of many current fMRI-nf studies: to train neural regulation, modify behavior (Thibault et al., 2018), or reduce clinical symptoms (Jeunet et al., 2019). Several studies have shown that fMRI-nf can modulate the activity of motor-related areas, such as the primary motor cortex (M1; e.g., Blefari, Sulzer, Hepp-Reymond, Kollias, \& Gassert, 2015; Chiew, LaConte, \& Graham, 2012), supplementary motor area (SMA; e.g., Lafleur et al., 2002; Scharnowski et al., 2015), and premotor cortex (PMC; e.g., Johnson et al., 2012; Marins et al., 2015), and the strategies used to activate motor-related regions include motor execution (ME) and motor imagery (MI; Berman, Horovitz, Venkataraman, \& Hallett, 2012).

15 However, the brain network involved in motor is complex (Hanakawa, 2016), and the difference in the modulatory effects of NF on different motor-related regions is still unclear. If the effects in motor-related regions are similar, researchers can feed information from any of these regions back to participants, regardless of the region they are primarily interested in, so it is valuable to compare different neurofeedback modulatory effects 20 between different motor-related regions. Mehler (2019) asked participants to increase the M1 activity in five neurofeedback runs (M1 condition) and to increase SMA activity in five separate neurofeedback runs (SMA condition), and they found that neither M1 nor SMA activity showed a statistically significant difference between the two conditions. However, 
Mehler (2019) aimed to compare the ability to self-regulate the M1 versus SMA among participants, while the inter-participant modulatory effects of motor-related areas using the same experimental protocol remain to be compared and are therefore unclear.

Thus, our study focused on comparing the modulatory effects of neurofeedback between two distinct motor-related regions: the left M1 and the left ventral premotor cortex (PMv). Here we categorized participants into two groups - those receiving feedback from either the left M1 or the left PMv. Both groups were given the same instructions. Therefore, any different treatment effects between the two groups would indicate differences between the modulatory effects of the two target regions. 


\section{Materials and Methods}

\section{Participants}

Thirty healthy volunteers [16 men; age (mean \pm SD), $23.9 \pm 3.07$ years] participated in the experiment. The sample size was decided according to a review study suggesting that the mean participants for one group in previous fMRI-nf studies was $13.7 \pm 7.35$ (mean $\pm \mathrm{SD}$; Thibault et al., 2018). All participants were right-handed, as assessed by the Japanese version of the Edinburgh Handedness Inventory (Oldfield, 1971; Japanese version by Hatta and Nakatsuka, 1975). The experimental design received approval from the local ethics committee. Each participant provided written informed consent before the commencement of the study.

Participants were randomly assigned into the following two groups, matched by gender: M1 group received BOLD signals from their left M1 as feedback $(n=15)$, and the PMv-group received signals from their left PMv $(n=15)$.

\section{Task procedures}

The complete experimental protocol consisted of one practice period, six neurofeedback runs, and one questionnaire period (Figure 1A). Participants were instructed to attempt to increase the activity of their motor-related regions, and simultaneously perform hand movements or imagine hand movements in the execution or imagery condition, respectively. During the practice period, participants learned to clench and then release their right hand every time the number presented on the screen counted backward by $2 \mathrm{~s}$ from 10 (see feedback period in Figure 1B). The execution and imagery conditions were alternatively performed over six neurofeedback runs, with three runs per condition (Figure 1A). In both 
conditions, each run first included $20 \mathrm{~s}$ to reach the T1 steady state, followed by eight blocks alternating between 20 -s baseline and 20 -s feedback periods. During the baseline periods, a fixation cross was presented on the screen. During the feedback periods, two horizontal line-segments and a white solid circle were presented on the screen (Figure 1C).

5 The distance of the red line-segment from the solid circle represented $1 \%$ increase in the BOLD signal from the average signal intensity during the baseline period. The green line-segment was updated every $2 \mathrm{~s}$ (Figure 1B), and its distance from the solid circle was calculated from the activity of the participant's target region at that time.

FIGURE 1 ABOUT HERE

Participants were instructed to try to increase the height of the green line to reach the red line. We told participants that execution and imagery of hand movements could help them increase the height of the green line because it was calculated from their motor-related brain region. They were also instructed to find an effective way to increase their brain activity using the feedback information. In the imagery condition, participants were asked to do the same task as in the execution condition, except that they had to imagine clenching their right fist every $2 \mathrm{~s}$ instead of performing clenching. After the participants completed all MR scans, they were asked about the strategies they used in the neurofeedback period. This helped rate the sense of control (controllability) during execution runs and imagery runs on a 10-point scale, with higher scores corresponding to a better sense of control.

All participants were given the same instructions before the practice stage, regardless of the group. Besides, they were informed of the delay in the feedback signal caused by the hemodynamic response and of relaxing as much as possible during the baseline period, because the height of the green line indicated the comparable increase from baseline 
duration. After the experiment was completed, they received a debriefing about the aim of this experiment and from which part of the brain the feedback came from.

\section{MRI acquisition}

5 Images were taken using a Siemens (Erlangen, Germany) 3-Tesla Prisma scanner with a 64-channel head coil at the Research and Education Center for Brain Science, Hokkaido University. BOLD signals were measured using echo-planar imaging sequence (volume repetition time, $2 \mathrm{~s}$; echo time, $30 \mathrm{~ms}$; flip angle, $90^{\circ}$ ). The entire brain was covered in 35 axial slices paralleled to the AC-PC line, with a voxel size of $3 \times 3 \times 3 \mathrm{~mm}^{3}$. The first three scans of each run were discarded to avoid T1 equilibration effects. T1-weighted structural images covering the whole brain were acquired with a resolution of $0.83 \times 0.83 \times 0.8-\mathrm{mm}^{3}$ after acquiring functional images using a T1 MP-RAGE sequence $(\mathrm{TR}=2300 \mathrm{~ms}, \mathrm{TE}=$ $2.41 \mathrm{~ms}, \mathrm{TI}=900 \mathrm{~ms}, \mathrm{FOV}=256 \times 256 \mathrm{~mm}^{2}$, and an $8^{\circ}$ flip angle).

\section{Online data analysis}

The functional data acquired from our experiments were exported to a shared destination folder within the local network assessed by OpenNFT software via TCP/IP. We used OpenNFT (opennft.org; Koush et al., 2017), an open-source software framework, to process online fMRI data and to present the stimuli. A cumulative GLM was used to correct

20 linear drift and head motions in real-time, and an extension of a low-pass Kalman filter was applied to deal with high-frequency noise, and a first-order autoregressive model was used to deal with neurophysiological fluctuations (Koush et al., 2017).

Regions of interest (ROIs; the left M1 for the M1-group and the left PMv for the 
PMv-group) were identified in the functional images of individual participants' brains using a deformation module of SPM8 (Megumi et al., 2015). The template ROI files were defined in a standard brain (Mayka et al., 2006). Participants' ROI voxels were individually identified using the first scanned image acquired before the first execution run.

ROI activity levels (NF-Scores) were computed as a percentage of the average signal during the feedback period compared to the average during the baseline period directly preceding the feedback period. Sepulveda et al. (2016) suggested that reinforcing motivation could help brain self-regulation, and our preliminary experiment showed that participants felt frustrated when they were continuously receiving poor feedback. To prevent participants from feeling discouraged, we modified all negative NF-Scores to zero. Scores participants received were referred to as NF-Score, and the original scores were referred to as raw NF-Score. Since raw NF-Score reflected real-time brain activity, and the current study aimed to investigate whether the effects of different target regions differ in brain regulation, we analyzed raw NF-Score.

\section{Off-line data analysis}

Data analyses of self-reported controllability and NF-Scores were performed using MATLAB (MATLAB and Statistics Toolbox Release 2016b, The MathWorks, Inc., Natick, Massachusetts, United States). NF-score of PMv (PMv-Score) for the M1-group and M1 (M1-Score) for the PMv-group were also calculated using the same algorithm of OpenNFT.

Brain imaging analyses were performed using the SPM12 statistical parametric mapping software package (Wellcome Department of Cognitive Neurology, http://www.fil.ion.ucl.ac.uk/spm). The preprocessing included motion correction, 
realignment, co-registration, normalization to Montreal Neurological Institute (MNI) space, and smoothing with a Gaussian kernel with 6 mm full-width-at-half-maximum (FWHM). Using the general linear model (GLM), the execution and imagery condition were modeled as two separate regressors that were convolved with a canonical hemodynamic response 5 function for each participant. Motion parameters were used as covariates to capture residual motion artifacts. Low-frequency noise was removed using a high-pass filter with a cut-off period of $128 \mathrm{~s}$. Contrasts were generated between each condition and the baseline and between the execution and the imagery for each participant. A random-effects model of a one-sample $t$-test was then used for group analysis. The significantly activated regions were reported with a threshold at $p<0.05$ corrected for family-wise error (FWE) using three-dimensional random field theory or with a threshold at $p<0.001$ uncorrected for multiple comparisons. Activated clusters were considered significant if their spatial extent was greater than 15 voxels. 


\section{Results}

\section{Self-reported Strategies and Controllability}

All participants reported that they were moving their hands during the execution runs and trying to increase the NF-Score by changing the power of hand movement. However, the strategies used in imagery runs were not limited to the imagery of the right-hand clenching every $2 \mathrm{~s}$. Most participants explained that they changed strategies during the imagery condition because they failed to control the NF-Score by imagining right-hand clenching at the beginning of the neurofeedback period. To increase their NF-Score, they kept trying different strategies. For example, they imagined doing more complex actions with their right hand (e.g., playing tennis and writing) or clenching their hands while focusing on some trivial sensation (e.g., the movement of a small part of the muscle or one of the knuckles).

A mixed-design analysis of variance (ANOVA) was used to compare the effects of group (M1-group and PMv-group) as a between-subjects factor and condition (execution and imagery) as a within-subjects factor on participants' self-reported controllability (Figure 2A). The ANOVA revealed a significant main effect on condition $(F(1,28)=17.7, p<.001$, $\left.\eta_{\mathrm{p}}{ }^{2}=0.39\right)$ and a marginally significant interaction effect between groups and conditions $\left(F(1,28)=3.67, p=.066, \eta_{\mathrm{p}}^{2}=.12\right)$. Post hoc comparisons using the Holm-Bonferroni method showed that the simple effect of condition in the M1-group was significant $(F(1,14)$ ${ }_{20}=28.33, p<.001$ ), whereas condition effects in the PMv-group were not significant $(F(1,14)=2, p=.18)$. We did not observe any difference between the groups in terms of execution condition $(F(1,28)=0.43, p=.52)$ or imagery condition $(F(1,28)=2.4, p=.13)$. These results indicated that the task condition had a significant effect on the self-reported 
controllability in the M1-group but not in the PMv-group.

FIGURE 2 ABOUT HERE

\section{NF-Score Regulation}

5 Averaged NF-Scores participants received were presented in Figure 2B, showing that M1-group was presented with visible higher NF-Scores during the execution condition.

A one-sample $t$-test was conducted to determine if a difference existed between the brain activity during the feedback period and the baseline period by comparing raw NF-Scores with zero. The significance level for Student's $t$-test was $0.00625(0.05 / 8)$, adjusted using a

Bonferroni correction. For execution condition, both M1-Score and PMv-Score for both M1-group and PMv-group were significantly greater than zero (see details in Table 1 and Figure 2C \& 2D). However, for imagery condition, the PMv-Score for the PMv-group was significantly greater than zero $(t(14)=3.69, p=.002$, Cohen's $d=0.953)$, and the M1-Score for the PMv-group was marginally significantly less than zero $(t(14)=-2.99, p$ $15=.01$, Cohen's $d=-0.773$ ).

An independent-samples $t$-test was then conducted to compare M1/PMv-Scores in M1-group and PMv-group. The results revealed no significant differences (see details in Table 2).

To investigate the effects of group and condition on the raw NF-Score (M1-Score for M1-group and PMv-Score for PMv-group), a mixed-designed ANOVA was performed. The main effect of group $\left(F(1,28)=8.197, p=.008, \eta_{p}^{2}=.226\right)$, the main effect of condition $\left(F(1,28)=132.003, p<.001, \eta_{\mathrm{p}}^{2}=.825\right)$, and the interaction effect $(F(1,28)=$ 
109.204, $\left.p<.001, \eta_{\mathrm{p}}{ }^{2}=.796\right)$ were significant. Post hoc comparison using the t-test with Bonferroni correction showed that $\mathrm{M} 1$-group/execution $(\mathrm{M}=19.4, \mathrm{SD}=4.239)$ was significantly different from $\mathrm{M} 1$-group/imagery $(\mathrm{M}=-1.893, \mathrm{SD}=6.529)$, and further, PMv-group/execution $(\mathrm{M}=5.019, \mathrm{SD}=3.983)$ and $\mathrm{M} 1$-group/imagery was significantly

5 different from PMv-group/imagery $(\mathrm{M}=4.01, \quad \mathrm{SD}=4.208)$, while the PMv-group/execution did not significantly differ from the PMv-group/imagery.

A two-way repeated-measures ANOVA was conducted to examine the effect of condition and time (three runs each condition) on each participant's average NF-Score. The result suggested that for the NF-Scores in the M1-group, the main effect of the condition was significant $\left(F(2,28)=174.247, p<.001, \eta_{\mathrm{p}}^{2}=.926\right.$; Figure 3A $)$. While in the PMv-group, NF-Score in the execution runs tended to increase, whereas those in the imagery runs tended to decrease, which was reflected in the significant interaction effect of conditions and runs $\left(F(2,28)=3.57, p=.042, \eta_{\mathrm{p}}{ }^{2}=.203\right.$; Figure 3B $)$. No other main effects or interactions were significant $\left(F(2,28) \leq 1.478, p \geq .245, \eta_{\mathrm{p}}{ }^{2} \leq .096\right)$.

\section{fMRI Mass-Univariate Analysis}

Regions activated significantly for all participants during execution and imagery periods compared with the baseline were reported because the analyses showed no difference between the groups. These comparisons revealed most of the activation in the lateral cerebellum, left postcentral gyrus, right opercular part of the inferior frontal gyrus, and right inferior parietal lobule during the execution condition, and the right precentral cortex, left SMA, left Rolandic operculum, and right inferior parietal lobule during the imagery 
condition (Figure 4). Subsequently, we analyzed the contrasts (execution versus imagery) in both groups. Increased activation in the right cerebellum and left postcentral cortex in both groups were observed during execution compared with that during imagery. Also, the contrast of imagery versus execution showed selective activation in the right precentral cortex (cluster size, $k=51$ ) in the PMv-group, while no significant differences were observed in the M1-group. 


\section{Discussion}

The primary aim of this study was to compare the modulatory effects of neurofeedback from different motor-related regions — the left M1 and the left PMv. Our results showed that during the imagery condition, the M1-Score was marginally significantly less than zero in the PMv-group but was not in the M1-group. This result suggested that the left M1 was deactivated in the PMv-group but was not in the M1-group, indicating that the M1-group regulated their left M1 activity in a positive direction. Furthermore, the PMv-Score was significantly greater than zero in the PMv-group but was not in the M1-group, indicating that PMv-group succeeded in increasing their PMv activity while M1-group did not. Consider that participants in both groups were provided with the same instructions, were performing the same tasks, and were receiving real and task-related neurofeedback from their own brain, our results indicated that neurofeedback rather than the mental task, i.e., motor imagery in this study, has effects on brain activity regulation and neurofeedback from different motor-related regions has a different impact on the brain regulation.

15 Moreover, NF-Scores showed a significant interaction effect over conditions and runs in the PMv-group. Previous studies have indicated that PMv plays a crucial role in hand movements (e.g., Rizzolatti, Fogassi, \& Gallese, 2002) and motor imagery (e.g., Binkofski et al., 2000). Moreover, greater activation of PMv during imagery versus execution condition (Guillot et al., 2008), and the increase of premotor activation after a one-week physical practice of hand movements (Lacourse et al., 2005) were found. We thus hypothesized the change of PMv-Scores during neurofeedback training would show an interaction effect between conditions. Our study suggested that the trends of PMv-Scores were different between conditions, indicating that the role played by $\mathrm{PMv}$ during the 
neurofeedback training process in different conditions is different.

The result that participants were able to activate the left M1 during execution runs is congruent with previous studies investigating the modulatory effect of neurofeedback using motor-related cortex as target region to facilitate the hand motor task performance (Berman

5 et al., 2012; DeCharms et al., 2004). Furthermore, fMRI univariate analysis suggests that the right $\mathrm{M} 1$ of the PMv-group during the execution condition was deactivated compared with that during the imagery condition. This result can be explained by the interhemispheric interactions between left M1 and right M1 that increased M1 activation in one hemisphere induces reduced M1 activation in the opposite hemisphere (Hamzei et al., 2002; Newton et al., 2005). Moreover, the result that participants could not activate the left M1 during imagery runs is congruent with a meta-analysis study of motor imagery (Hétu et al., 2013), suggesting motor imagery did not consistently activate contralateral M1. Since our results showed that the activity of left M1 is related to motor execution but not motor imagery, as Mehler et al. (2019) suggested, contralateral M1 is not an evaluable option for motor imagery-based fMRI neurofeedback training.

The limitation of the current study is the sample size. A sample size of 84 participants per group is needed to detect a difference between the two independent groups with $80 \%$ power, using a two-sample $t$-test and assuming a two-sided $\alpha$ of 0.05 and an effect size $d$ of 0.386 (see Table 2), calculated by using G*Power3 (Faul, F., Erdfelder, E., Lang, A. G., \& Buchner, 2007). Thus, we cannot conclude that there was no difference between the two groups under a sample size of 15 participants.

In summary, we found left M1 was deactivated in the PMv-group but not in the M1-group, whereas the left PMv was activated in the PMv-group but not in the M1-group during 
imagery condition. Moreover, only in the PMv-group, raw NF-Scores showed a significant interaction effect over conditions and runs. These results indicated that neurofeedback from distinct motor-related regions has different effects on brain activity regulation.

\section{Acknowledgments}

The authors would like to thank Enago (www.enago.jp) for the English language review.

\section{Funding}

This work was supported by JSPS KAKENHI Grant Number 17H04683 and Graduate 


\section{References}

Berman, B.D., Horovitz, S.G., Venkataraman, G., Hallett, M., 2012. Self-modulation of primary motor cortex activity with motor and motor imagery tasks using real-time fMRI-based neurofeedback. Neuroimage. https://doi.org/10.1016/j.neuroimage.2011.07.035

Binkofski, F., Amunts, K., Stephan, K.M., Posse, S., Schormann, T., Freund, H.J., Zilles, K., Seitz, R.J., 2000. Broca's region subserves imagery of motion: A combined cytoarchitectonic and fMRI study. Hum. Brain Mapp. 11, 273-285. https://doi.org/10.1002/1097-0193(200012)11:4<273::AID-HBM40>3.0.CO;2-0

Blefari, M.L., Sulzer, J., Hepp-Reymond, M.-C., Kollias, S., Gassert, R., 2015. Improvement in precision grip force control with self-modulation of primary motor cortex during motor imagery. Front. Behav. Neurosci. 9, 1-11. https://doi.org/10.3389/fnbeh.2015.00018

Chiew, M., LaConte, S.M., Graham, S.J., 2012. Investigation of fMRI neurofeedback of differential primary motor cortex activity using kinesthetic motor imagery. Neuroimage. https://doi.org/10.1016/j.neuroimage.2012.02.053

DeCharms, R.C., Christoff, K., Glover, G.H., Pauly, J.M., Whitfield, S., Gabrieli, J.D.E., 2004. Learned regulation of spatially localized brain activation using real-time fMRI. Neuroimage 21, 436-443. https://doi.org/10.1016/j.neuroimage.2003.08.041 statistical power analysis program for the social, behavioral, and biomedical sciences $39,175-191$.

Guillot, A., Collet, C., Nguyen, V.A., Malouin, F., Richards, C., Doyon, J., 2008. 
Functional neuroanatomical networks associated with expertise in motor imagery. Neuroimage 41, 1471-1483. https://doi.org/10.1016/j.neuroimage.2008.03.042

Hamzei, F., Dettmers, C., Rzanny, R., Liepert, J., Büchel, C., Weiller, C., 2002. Reduction of excitability ("inhibition") in the ipsilateral primary motor cortex is mirrored by fMRI signal decreases. Neuroimage 17, 490-496.

https://doi.org/10.1006/nimg.2002.1077

Hanakawa, T., 2016. Organizing motor imageries. Neurosci. Res. 104, 56-63. https://doi.org/10.1016/j.neures.2015.11.003

Hétu, S., Grégoire, M., Saimpont, A., Coll, M.P., Eugène, F., Michon, P.E., Jackson, P.L., 2013. The neural network of motor imagery: An ALE meta-analysis. Neurosci. Biobehav. Rev. 37, 930-949. https://doi.org/10.1016/j.neubiorev.2013.03.017

Jeunet, C., Glize, B., McGonigal, A., Batail, J.M., Micoulaud-Franchi, J.A., 2019. Using EEG-based brain computer interface and neurofeedback targeting sensorimotor rhythms to improve motor skills: Theoretical background, applications and prospects. Neurophysiol. Clin. 49, 125-136. https://doi.org/10.1016/j.neucli.2018.10.068

Johnson, K.A., Hartwell, K., Lematty, T., Borckardt, J., Morgan, P.S., Govindarajan, K., Brady, K., George, M.S., 2012. Intermittent "Real-time” fMRI Feedback Is Superior to Continuous Presentation for a Motor Imagery Task: A Pilot Study. J. Neuroimaging 22, 58-66. https://doi.org/10.1111/j.1552-6569.2010.00529.x

Koush, Y., Ashburner, J., Prilepin, E., Sladky, R., Zeidman, P., Bibikov, S., Scharnowski, F., Nikonorov, A., Ville, D. Van De, 2017. NeuroImage OpenNFT : An open-source Python / Matlab framework for real-time fMRI neurofeedback training based on activity, connectivity and multivariate pattern analysis. Neuroimage 156, 489-503. 
https://doi.org/10.1016/j.neuroimage.2017.06.039

Lacourse, M.G., Orr, E.L.R., Cramer, S.C., Cohen, M.J., 2005. Brain activation during execution and motor imagery of novel and skilled sequential hand movements. Neuroimage 27, 505-519. https://doi.org/10.1016/j.neuroimage.2005.04.025

5 Lafleur, M.F., Jackson, P.L., Malouin, F., Richards, C.L., Evans, A.C., Doyon, J., 2002. Motor learning produces parallel dynamic functional changes during the execution and imagination of sequential foot movements. Neuroimage 16, 142-157. https://doi.org/10.1006/nimg.2001.1048

Marins, T.F., Rodrigues, E.C., Engel, A., Hoefle, S., Basilio, R., Lent, R., Moll, J., Tovar-Moll, F., 2015. Enhancing Motor Network Activity Using Real-Time Functional MRI Neurofeedback of Left Premotor Cortex. Front Behav Neurosci 9, 341. https://doi.org/10.3389/fnbeh.2015.00341

Mayka, M.A., Corcos, D.M., Leurgans, S.E., Vaillancourt, D.E., 2006. Three-dimensional locations and boundaries of motor and premotor cortices as defined by functional brain imaging: A meta-analysis. Neuroimage 31, 1453-1474. https://doi.org/10.1016/j.neuroimage.2006.02.004

Megumi, F., Yamashita, A., Kawato, M., Imamizu, H., 2015. Functional MRI neurofeedback training on connectivity between two regions induces long-lasting changes in intrinsic functional network. Front Hum Neurosci 9, 160. https://doi.org/10.3389/fnhum.2015.00160

Mehler, D.M.A., Williams, A.N., Krause, F., Lührs, M., Wise, R.G., Turner, D.L., Linden, D.E.J., Whittaker, J.R., 2019. The BOLD response in primary motor cortex and supplementary motor area during kinesthetic motor imagery based graded fMRI 
neurofeedback. Neuroimage 184, 36-44.

https://doi.org/10.1016/j.neuroimage.2018.09.007

Newton, J.M., Sunderland, A., Gowland, P.A., 2005. fMRI signal decreases in ipsilateral primary motor cortex during unilateral hand movements are related to duration and side of movement. Neuroimage 24, 1080-1087.

https://doi.org/10.1016/j.neuroimage.2004.10.003

Rizzolatti, G., Fogassi, L., Gallese, V., 2002. Motor and cognitive functions of the ventral premotor cortex. Current opinion in neurobiology, 12(2), 149-154. 149-154. https://doi.org/10.1016/S0959-4388(02)00308-2.

Scharnowski, F., Veit, R., Zopf, R., Studer, P., Bock, S., Diedrichsen, J., Goebel, R., Mathiak, K., Birbaumer, N., Weiskopf, N., 2015. Manipulating motor performance and memory through real-time fMRI neurofeedback. Biol. Psychol. 108, 85-97. https://doi.org/10.1016/j.biopsycho.2015.03.009

Sepulveda, P., Sitaram, R., Rana, M., Montalba, C., Tejos, C., Ruiz, S., 2016. How feedback, motor imagery, and reward influence brain self-regulation using real-time fMRI. Hum. Brain Mapp. 37, 3153-3171. https://doi.org/10.1002/hbm.23228

Sulzer, J., Haller, S., Scharnowski, F., Weiskopf, N., Birbaumer, N., Blefari, M.L., Bruehl, A.B., Cohen, L.G., deCharms, R.C., Gassert, R., Goebel, R., Herwig, U., LaConte, S., Linden, D., Luft, A., Seifritz, E., Sitaram, R., 2013. Real-time fMRI neurofeedback: Progress and challenges. Neuroimage 76, 386-399. https://doi.org/10.1016/j.neuroimage.2013.03.033

Thibault, R.T., MacPherson, A., Lifshitz, M., Roth, R.R., Raz, A., 2018. Neurofeedback with fMRI: A critical systematic review. Neuroimage 172, 786-807. 
https://doi.org/10.1016/j.neuroimage.2017.12.071 


\section{Figures}

A

\begin{tabular}{|c|}
\hline Practice \\
\hline Execution Run 1 \\
\hline Imagery Run 1 \\
\hline Execution Run 2 \\
\hline Imagery Run 2 \\
\hline Execution Run 3 \\
\hline Imagery Run 3 \\
\hline Questionnaire \\
\hline
\end{tabular}

B
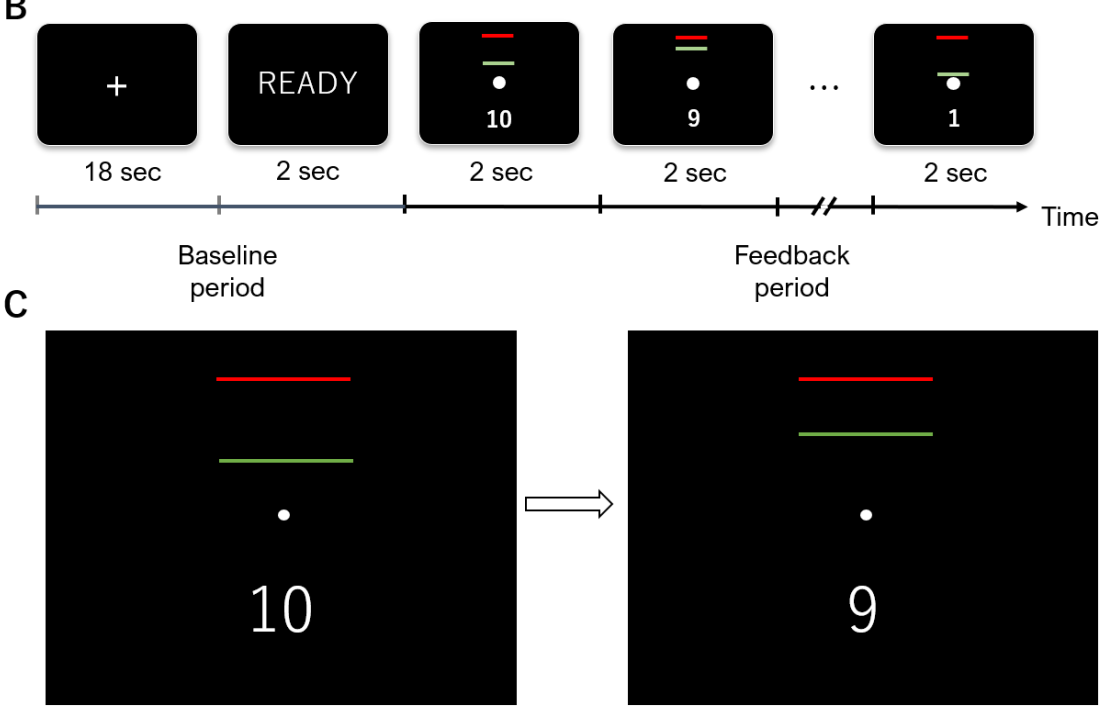

Figure 1:

The procedure of the main experiment. A) Stages of the experiment. B) The example

5 timeline of one block in the neurofeedback runs. Each block consisted of a 20-s baseline period and a 20 -s feedback period. During the feedback period, the screen was updated every 2 s. C) The screen during the feedback period, with a solid white circle, a red target line-segment, a green neurofeedback line-segment, and a number showing how many sets of hand movements were left in this block. 
Figure 2:

A
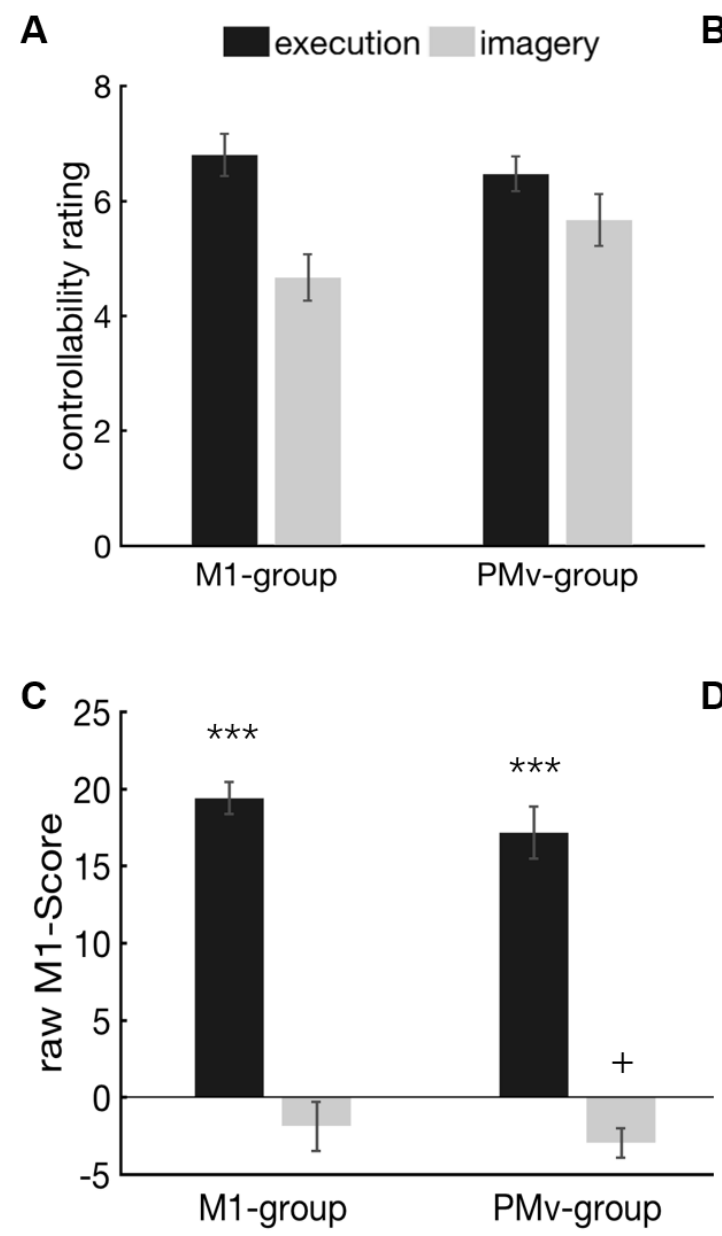

B
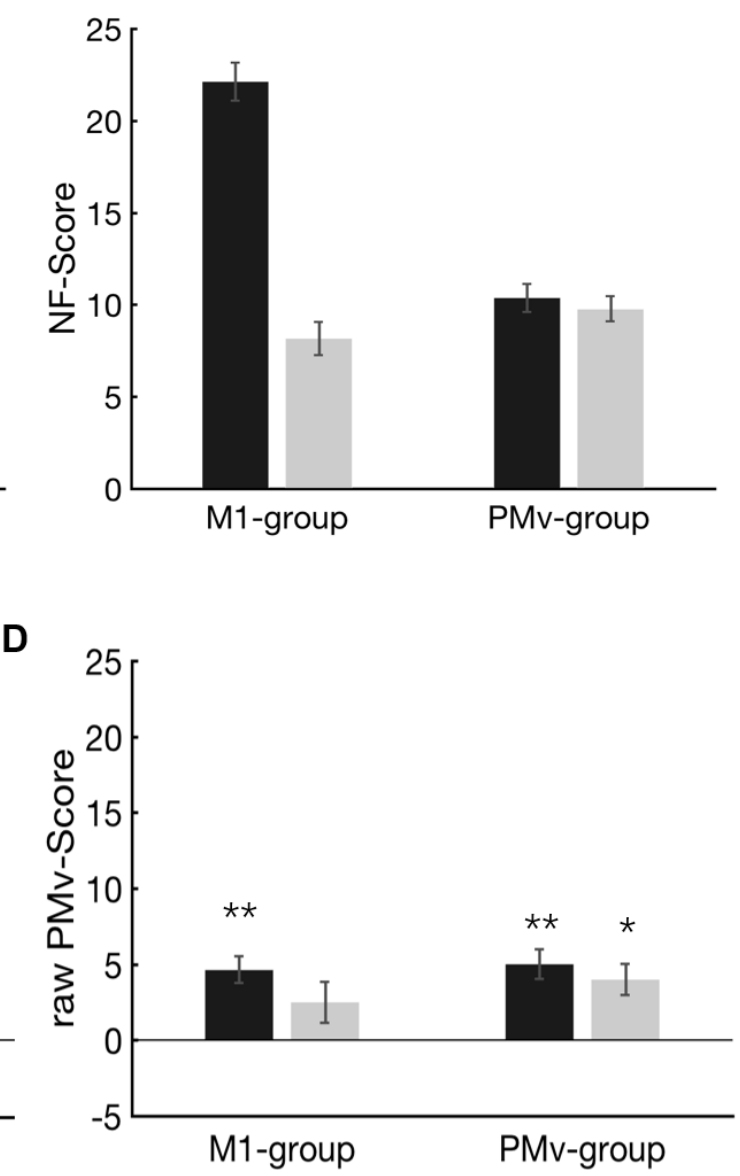

Averaged scores of the two groups. A) Averaged self-reported controllability of M1-group and PMv-group during the execution and imagery conditions. B) Averaged NF-Score that 5 participants received during the execution and imagery conditions. C) Averaged raw M1-Score during the execution and imagery conditions. D) Averaged raw PMv-Score during the execution and imagery conditions. Error bars denote the standard error of the mean. $+: p<.125, *: p<0.00625, * *: p<0.00125, * * *: p<0.00013$. 
Figure 3:
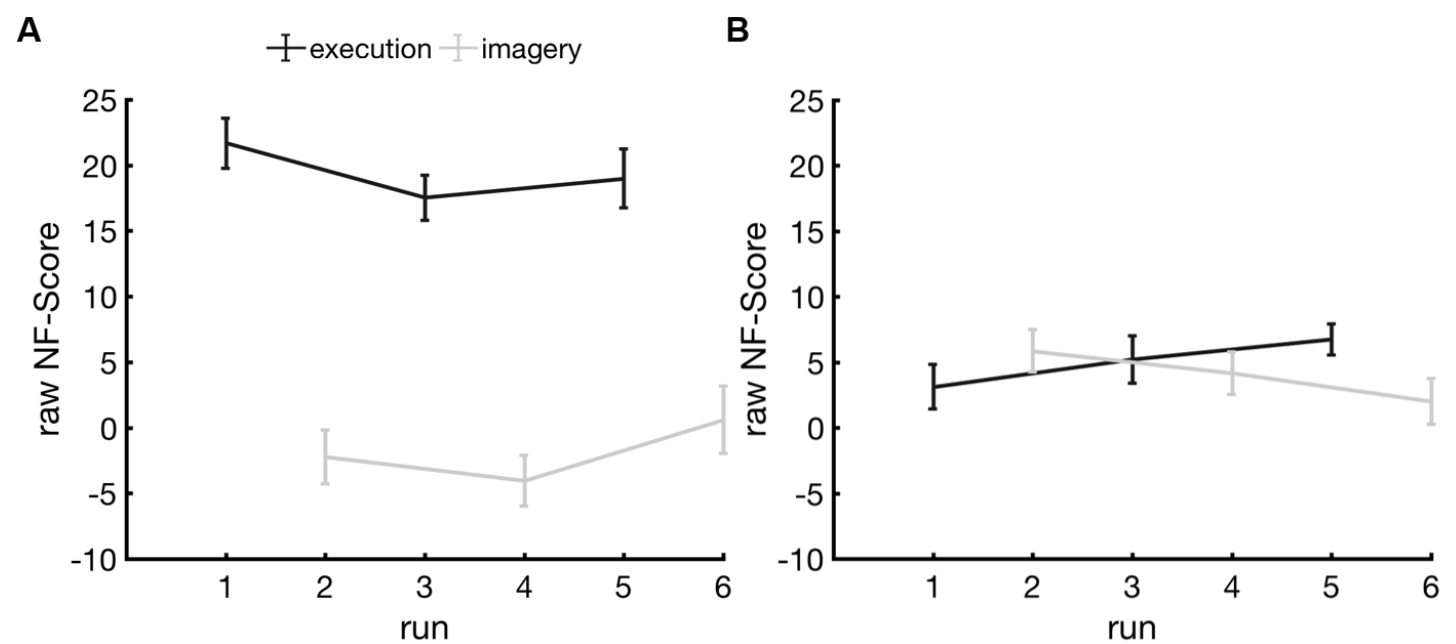

Averaged time series. A) Averaged raw NF-Scores of the M1-group across runs; B) Averaged raw NF-Scores of the PMv-group across runs. 


\section{Figure 4:}

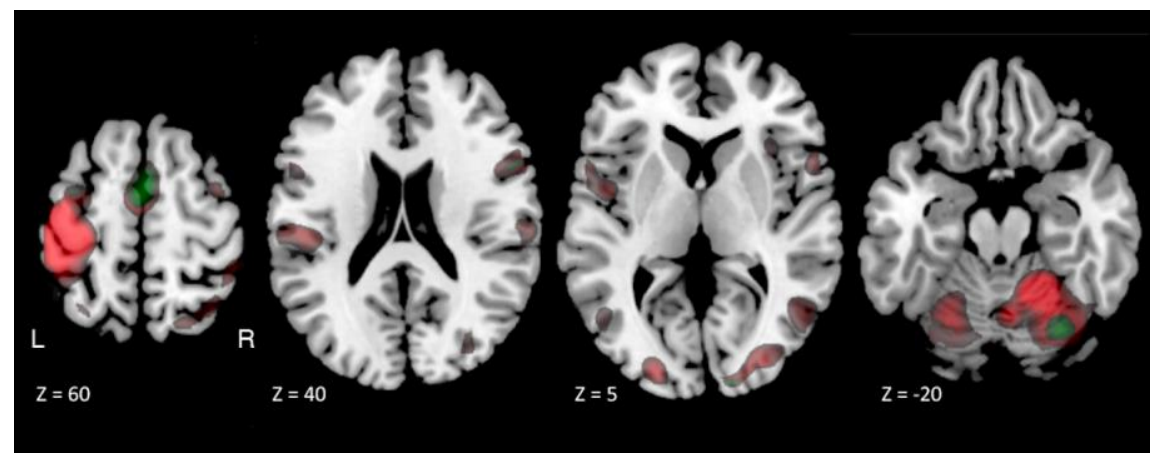

fMRI univariate analysis results. Red: Areas activated in the motor execution condition.

Green: Areas activated in the motor imagery condition. Activation was reported with a 5 threshold of $p<0.05$ corrected for multiple comparisons for family-wise error (FWE) with an extent threshold of 15 voxels. L, left hemisphere; R, right hemisphere. Figures are displayed in the horizontal plane with $\mathrm{Z}$ denoting locations in the MNI coordinates. 
Tables

Table 1: Two-tailed one-sample $t$-test results for raw M1-Score and PMv-Score for M1-group and PMv-group.

\begin{tabular}{|c|c|c|c|c|c|c|c|c|}
\hline NF-Score & group & condition & Mean & SD & $t(14)$ & $p$ & & $\begin{array}{r}\text { Cohen's } \\
d\end{array}$ \\
\hline \multirow{4}{*}{$\begin{array}{l}\text { M1- } \\
\text { Score }\end{array}$} & \multirow{2}{*}{$\begin{array}{l}\text { M1- } \\
\text { group }\end{array}$} & Execution & 19.408 & 4.239 & 17.732 & $<.00013$ & $* * *$ & 4.578 \\
\hline & & Imagery & -1.893 & 6.529 & -1.123 & 0.28 & & -0.29 \\
\hline & \multirow{2}{*}{$\begin{array}{l}\text { PMv- } \\
\text { group }\end{array}$} & Execution & 17.172 & 7.007 & 9.491 & $<.00013$ & $* * *$ & 2.451 \\
\hline & & Imagery & -2.987 & 3.866 & -2.992 & 0.01 & + & -0.773 \\
\hline \multirow{4}{*}{$\begin{array}{l}\text { PMv- } \\
\text { Score }\end{array}$} & \multirow{2}{*}{$\begin{array}{l}\text { M1- } \\
\text { group }\end{array}$} & Execution & 4.641 & 3.751 & 4.792 & $<.00125$ & $* *$ & 1.237 \\
\hline & & Imagery & 2.511 & 5.569 & 1.747 & 0.103 & & 0.451 \\
\hline & \multirow{2}{*}{$\begin{array}{l}\text { PMv- } \\
\text { group }\end{array}$} & Execution & 5.019 & 3.983 & 4.88 & $<.00125$ & $* *$ & 1.26 \\
\hline & & Imagery & 4.01 & 4.208 & 3.691 & 0.002 & $*$ & 0.953 \\
\hline
\end{tabular}


Table 2: Two-tailed independent samples $t$-test results for the effect of group on the raw M1-Score and PMv-Score during execution and imagery conditions.

\begin{tabular}{cl|rrr}
\hline NF-Score & condition & $\boldsymbol{t}(\mathbf{2 8 )}$ & $\boldsymbol{p}$ & Cohen's $\boldsymbol{d}$ \\
\hline M1- & Execution & 1.057 & 0.299 & 0.386 \\
Score & Imagery & 0.558 & 0.581 & 0.204 \\
PMv- & Execution & -0.268 & 0.791 & -0.098 \\
Score & Imagery & -0.832 & 0.413 & -0.304 \\
\hline
\end{tabular}

